VOL. 51 (1995) [489-494]

\title{
TOTALLY DISCONNECTED GROUPS AND PROOFS OF CONJECTURES OF HOFMANN AND MUKHERJEA
}

\author{
GEORGE WILLIS
}

\begin{abstract}
Some structure theorems for totally disconnected groups are described. These theorems produce a certain positive integer valued function, called the scale function, on each totally disconnected group. The scale function has properties connected to the structure of the group and can be used to prove some conjectures of Hofmann and Mukherjea.
\end{abstract}

\section{INTRODUCTION}

One of the first facts proved about general locally compact groups is that each such group, $G$, is an extension

$$
\{e\} \rightarrow G_{0} \rightarrow G \rightarrow G / G_{0} \rightarrow\{e\}
$$

where $G_{0}$ is the connected component of $G$ containing the identity element, $e$, and $G / G_{0}$ is a totally disconnected group, see $[10,3,9]$. The study of locally compact groups thus divides into the study of these connected and totally disconnected factors.

A connected group, $C$, can be 'approximated' by Lie groups in the sense that each neighbourhood of $e$ contains a compact, normal subgroup, $K$, such that $C / K$ is a connected Lie group. The study of connected groups thus reduces to being essentially just the study of Lie groups. This analysis of the structure of connected groups was stimulated by Hilbert's fifth problem, of which it is the solution, see $[\mathbf{8}, 9,6]$.

The theory of general totally disconnected groups is much less well developed than that of connected groups. Almost the only progress which has been made is the theorem of van Dantzig, [12], that each totally disconnected group has a compact, open subgroup, also see $[1,3,9]$. It has not been at all clear what form a detailed structure theory for this class of groups would take and, in consequence, there have been few conjectures made about them. Indeed, it has been the commonly held opinion that there is no structure to be elucidated, for example see [1], historical note after Chapter III.

Received 4th August, 1994.

I wish to thank Professor J. M. Rosenblatt for several stimulating conversions and for drawing my attention to the paper [5] and Professor K. H. Hofmann for the research report [4].

Copyright Clearance Centre, Inc. Serial-fee code: 0004-9729/95 \$A2.00+0.00. 
Hofmann and Mukherjea conjectured, in [5], that locally compact groups cannot exhibit a certain strange property and, by means of an approximation by Lie groups argument, reduced their conjecture to the totally disconnected case. The results announced here were stimulated by this conjecture and may provide the beginning of a structure theory for totally disconnected groups. They suffice to prove the conjecture of Hofmann and Mukherjea and also another conjecture of Hofmann.

The structure theorems described here imply that there is a special integer valued function on each totally disconnected group. This function will be called the scale function. Much of the content of the theorems can be seen in the properties of this function. It is convenient to describe the scale function and its properties first; then to show how the scale function may be used to prove theorems about totally disconnected groups, including the conjectures of Hofmann and Mukherjea; and finally to state the structure theorems and explain how they produce the scale function.

\section{The SCALE Function}

The scale function is a continuous function, $s$, defined on each totally disconnected group, $G$, and taking positive integer values. It has the following properties.

I. For each $x$ in $G, s(x)=1=s\left(x^{-1}\right)$ if and only if $x$ normalises some compact, open subgroup of $G$.

Although, according to the theorem of van Dantzig, every totally disconnected group has a compact, open subgroup, they do not in general have compact, open, normal subgroups. An example which does not is the semidirect product group $H \times_{\alpha} \mathbb{Z}$, where $H=\left\{h=\left(h_{n}\right)_{n \in Z}: h_{n}=0\right.$ whenever $-n$ is sufficiently large $\}$ and $\alpha(h)_{n}=h_{n+1}$. It follows from $I$ that, if a group $G$ does have a compact, open, normal subgroup, then $s \equiv 1$. The converse fails however: it is not difficult to construct groups with $s \equiv 1$ but which do not have such normal subgroups.

II. For each $x$ in $G, \mathrm{~s}\left(x^{n}\right)=\mathrm{s}(x)^{n}, n \geqslant 1$.

It follows that, if $\mathbf{s}(x) \neq 1$, then $x$ cannot have roots of arbitrarily high order. Thus the scale function carries information about the existence of roots in the group. This observation provides a contrast with the theory of connected groups, as one of the key steps in that theory is to find group elements which are infinitely divisible.

The scale function is not multiplicative in general but it is closely related to a well known multiplicative function.

III. For each $x$ in $G, \Delta(x)=s(x) s\left(x^{-1}\right)^{-1}$, where $\Delta(x): G \rightarrow \mathbb{R}^{+}$is the modular function.

That the modular function on a totally disconnected group takes only rational values does not appear to be widely known even though it can be seen directly, that 
is, without recourse to the scale function, by considering the conjugates of an arbitrary compact, open subgroup.

IV. For each isomorphism, $\alpha: G \rightarrow B$ of totally disconnected groups and each $x$ in $G, \mathrm{~s}(\alpha(x))=\mathrm{s}(x)$.

\section{The conjectures of Hofmann and Mukherjea}

Hofmann and Mukherjea define a locally compact group to be strange if it is not compact and contains

(i) a cocompact, normal subgroup $H_{1}$,

(ii) a compact subgroup $H$ of $H_{1}$, and

(iii) an element $z$

such that for every neighbourhood $V$ of $H$, we have

$$
H_{1} \subset \operatorname{closure}\left(\bigcup_{n=1}^{\infty} z^{n} V z^{-n}\right) \text {. }
$$

This property arises in connection with a problem to do with the convolution powers of probability measures on $G$.

They show, in [5], that there are no strange connected groups. An important step in the proof is to use properties (ii) and (iii) to show that, in any complex representation $\pi$ of $G$, the spectrum of $\pi(h)$ is contained in the unit circle for every $h$ in $H_{1}$. Property (i) and a Lie group argument are then used to complete the proof.

In a joint paper with Rosenblatt, [11], it is shown that there are no strange totally disconnected groups. An important step in that proof is to use properties (ii) and (iii) to show that the scale function is identically 1 on $H_{1}$. Properties of the scale function are then used, together with (i), to complete the proof.

This suffices to prove the conjecture of Hofmann and Mukherjea.

THEOREM 1. There are no strange groups.

The scale function can also be used to answer another question of Hofmann. The question concerns the periodic elements in a group, where $x$ in $G$ is said to be periodic if the closed group it generates is compact. The set of all periodic elements in $G$ is denoted $P(G)$, see [7]. Hofmann asks, in [4], whether every totally disconnected group $G$ such that $P(G)$ is dense in $G$ in fact has $P(G)$ equal to $G$. (In the case of connected groups the answer is 'no' because the set of rotations and reflections is dense in the group of Euclidean motions of the plane. That translations can be approximated by rotations, which is what this amounts to, is attributed by Bourbaki to Descartes, [2], historical note to Chapter I.)

In the case of totally disconnected groups the answer is 'yes'. 
THEOREM 2. Let $G$ be a totally disconnected group. Then $P(G)$ is a closed subset of $G$.

Proof: Let $x$ be periodic and denote the closed subgroup $x$ generates by $\langle x\rangle^{-}$. Then, since $\langle x\rangle^{-}$is compact and $s$ is continuous, $s$ is bounded on $\langle x\rangle^{-}$. By II, $s$ is also multiplicative on $\langle x\rangle^{-}$, and so $s(x)=1$.

Now let $y$ be in the closure of $P(G)$. Then, since $\mathbf{s}$ is continuous, $\mathbf{s}(y)=1=$ $s\left(y^{-1}\right)$. Property $I$ of the scale function now implies that $y$ normalises some compact, open subgroup $U$. Since the set $U y$ is an open neighbourhood of $y$, there is $x$, periodic, in $U y$. Clearly, $x$ also normalises $U$ and so the group generated by $x$ and $U$ is just $U\langle x\rangle^{-}$, which is compact. This group contains the group generated by $y$. Therefore $y$ is periodic.

Here is another immediate consequence of the properties of the scale function, [13].

Theorem 3. Let $G$ be a totally disconnected group and $x$ be in $G$. Suppose there are $x_{n} \in G$ such that $\lim _{n \rightarrow \infty} x_{n}^{k_{n}}=x$, where the exponents $k_{n} \rightarrow \infty$ with $n$. Then $x$ normalises some compact, open subgroup of $G$.

Property IV of the scale function implies that it is an isomorphism invariant for totally disconnected groups. Therefore it can be used to distinguish between groups. For example, the values taken by the scale function on the automorphism group of the homogeneous tree of degree $n+1$ are computed in [13]. It is shown that an element $x$ is periodic if and only if $s(x)=1$ and that, otherwise, $s(x)=n^{k}$ where $k$ is the highest order of the roots of $x$. This provides a simple proof that the different automorphism groups are not isomorphic.

\section{Structure theorems and proof of existence of the scale function}

The existence of a continuous, integer valued function with the properties I IV follows from three structure theorems for totally disconnected groups. The first of these is a refinement of the theorem of van Dantzig. For each $x$ in $G$ it produces some compact, open subgroups which are well behaved under conjugation by $\boldsymbol{x}$.

THEOREM 4. Let $G$ be a totally disconnected group and $x$ be in $G$. Then there is a compact, open subgroup, $U$, of $G$ such that, putting $K_{+}=\bigcap_{n \geqslant 0} x^{n} U x^{-n}$ and $K_{-}=\bigcap_{n \geqslant 0} x^{-n} U x^{n}:$

(i) $U=K_{+} K_{-}=K_{-} K_{+}$

(ii) $\bigcup_{n \geqslant 0} x^{n} K_{+} x^{-n}$ is a closed subgroup of $G$.

Compact, open subgroups satisfying (i) and (ii) are said to be tidy for $x$. If $x$ normalises some compact, open subgroup, then that subgroup is tidy for $x$. Also, in the 
example $H \times_{\alpha} \mathbb{Z}$ mentioned above, the subgroup $H_{0}=\left\{\left(h_{n}\right) \in H: h_{n}=0\right.$ if $\left.n<0\right\}$ is tidy for $(0,1)$. In both of these examples the same subgroup is in fact tidy for every group element but in general the tidy subgroups vary with the element $x$. That $\bigcup_{n \geqslant 0} x^{n} K_{+} x^{-n}$ is a subgroup of $G$ follows immediately from its definition, the content of (ii) is that it is closed.

For an illustration of what tidiness means, consider the group $K \times_{\alpha} \mathbb{Z}$ where $K=\left\{k=\left(k_{n}\right)_{n \in Z}: k_{n} \in \mathbb{Z}_{2}\right\}$ is a compact infinite product group and $\alpha(k)_{n}=k_{n+1}$. $K$ is a compact, open, normal subgroup and so is tidy for $(0,1)$. On the other hand, the subgroup $V=\left\{k \in K: k_{-1}=0=k_{1}\right\}$ is not tidy because, using the notation of the theorem with $U$ replaced by $V, K_{+}=\left\{k \in K: k_{n}=0\right.$ for $\left.k \leqslant 1\right\}$ and $K_{-}=\{k \in K$ : $k_{n}=0$ for $\left.k \geqslant-1\right\}$ so that $K_{+} K_{-}=\left\{k \in K: k_{-1}=k_{0}=k_{1}=0\right\} \neq V$. If we next let $L=\left\{k \in K: k_{-1}=k_{0}=k_{1}=0\right\}$, then $K_{+}$and $K_{-}$are as before and so $L$ satisfies (i). However $\bigcup_{n \geqslant 0} x^{n} K_{+} x^{-n}=\left\{k \in K: k_{n}=0\right.$ whenever $-n$ is sufficiently large $\}$ and so $L$ does not satisfy (ii). The theorem is proved by taking an arbitrary compact, open subgroup and altering it to make it tidy for $x$. In the example just discussed, if $V$ were the group chosen these 'alterations' would first cut $V$ down to obtain the subgroup $L$ and then add what was required to recover the tidy subgroup $K$.

The second theorem permits the definition of the scale function.

ThEOREM 5. Suppose that $U^{(1)}$ and $U^{(2)}$ are tidy subgroups for $x$ and put

$$
K_{+}^{(i)}=\bigcap_{n \geqslant 0} x^{n} U^{(i)} x^{-n}, \quad i=1,2 .
$$

Then $\left[x K_{+}^{(1)} x^{-1}: K_{+}^{(1)}\right]=\left[x K_{+}^{(2)} x^{-1}: K_{+}^{(2)}\right]$ and this index is a positive integer.

A key step in the proof of this theorem is to show that the intersection of two tidy subgroups is tidy. The index $\left[x K_{+} x^{-1}: K_{+}\right]$is just the factor by which conjugation by $x$ scales up $K_{+}$and the value of the scale function at $x$ is defined to be this uniquely determined index. Thus, in the example $H \times_{\alpha} \mathbb{Z}, \mathbf{s}((0,1))=2$ and $\mathbf{s}((0,-1))=1$.

All properties of the scale function, apart from its continuity, follow from this definition and Theorems 1 and 2 . The continuity of $\mathbf{s}$ follows from the next theorem.

Theorem 6. Let $U$ be a tidy subgroup for $x$. Then $U$ is tidy for every $y$ in the double coset $U_{x} U$ and $\mathrm{s}(x)=\mathrm{s}(y)$.

\section{References}

[1] N. Bourbaki, General topology Part 1 (Herman, Paris, 1966).

[2] N. Bourbaki, Lie Groups and Lie Algebras (Springer-Verlag, Berlin, Heidelberg, New York, 1980). 
[3] E. Hewitt and K. A. Ross, Abstract Harmonic Analysis (Springer-Verlag, Berlin, Göttingen, Heidelberg, 1963 and 1970).

[4] K. H. Hofmann, 'Characteristic subgroups in locally compact totally disconnected groups and their applications to a problem on random walks on locally compact groups', Technische Hochschule Darmstadt, Fachbereich Mathematik Preprint Series 606 (1981), 1-12.

[5] K. H. Hofmann and A. Mukherjea, 'Concentration functions and a class of non-compact groups', Math. Ann. 256 (1981), 535-548.

[6] A. M. Gleason, 'Groups without small subgroups', Ann. of Math. 56 (1952), 193-212.

[7] S. Grosser and M. Moskowitz, 'Compactness conditions in topological groups', J. Reine Angew. Math. 246 (1971), 1-40.

[8] I. Kaplansky, Lie Algebras and Locally Compact Groups (Univ. Chicago Press, Chicago and London, 1974).

[9] D. Montgomery and L. Zippin, Topological Transformation Groups (Interscience Publishers, New York, 1955).

[10] L. Pontryagin, Topological groups, (English translation of 1939 Russian edition) (Princeton U.P., Princeton, 1946).

[11] J. M. Rosenblatt and G. A. Willis, 'Concentration functions in locally compact groups' (to appear).

[12] D. van Dantzig, 'Zur topologischen Algebra III. Brouwersche und Cantorsche Gruppen', Compositio Math. 3 (1936), 408-426.

[13] G. A. Willis, 'The structure of totally disconnected, locally compact groups', Math. Ann. (to appear).

Department of Mathematics

University of Newcastle

Newcastle, NSW 2308

Australia

e-mail: george@frey.newcastle.edu.au 